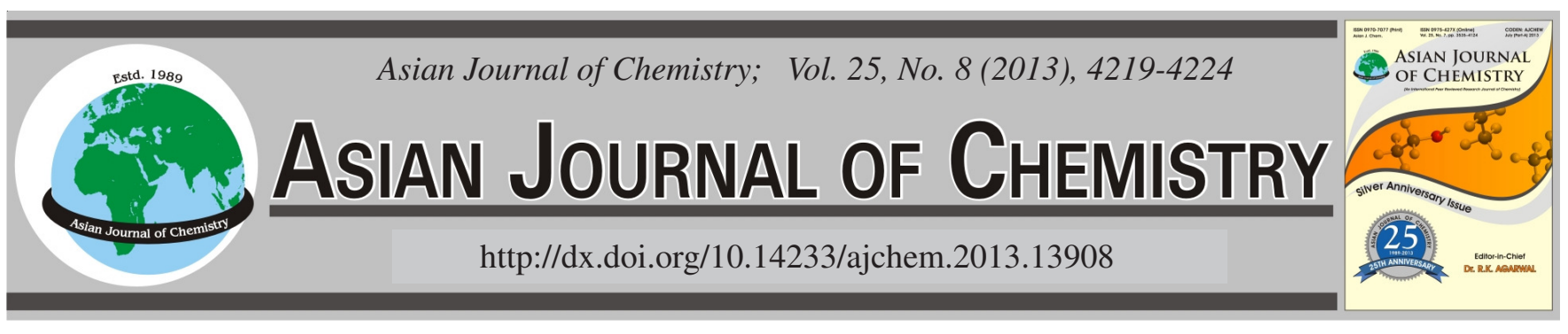

\title{
Synthesis of Organic Coagulant: Poly-diallyldimethyl Ammonium Chloride (PDADMAC) for Treatment of Sewage
}

\author{
Tiroyaone Tshukudu ${ }^{1,2}$, Huaili Z HenG $^{1,2, *}$ and Jun AnG $^{1,2}$
}

${ }^{1}$ Key Laboratory of the Three Gorges Reservoir Regions Eco-Environment, Ministry of Education, Chongqing University, Chongqing 400045, P.R. China

${ }^{2}$ Faculty of Urban Construction and Environmental Engineering, Chongqing University, Chongqing 400045, P.R. China

*Corresponding author: E-mail: zhl@cqu.edu.cn; zhl6512@126.com

\begin{abstract}
In this research, poly-diallyldimethyl ammonium chloride (PDADMAC) was synthesized using the Taguchi method of design of experiments and optimum synthesis conditions were determined. In addition, evaluation of treatment efficiency was determined by measuring both the reduction of residual turbidity and chemical oxygen demand (COD). The results show that the combination of factors adopted as optimum conditions using the Taguchi method were $50{ }^{\circ} \mathrm{C}, 0.15 \mathrm{~g}, 10 \mathrm{~mL}$ and $8 \mathrm{~h}$ for synthesis temperature, mass of the initiator, volume of deionized water and synthesis time, respectively. In addition, the ANOVA results indicate the synthesis temperature and the mass of the initiator played a key role on the synthesis of the PDADMAC. The optimal removal efficiency was achieved at coagulant dosage, $\mathrm{pH}$ value, flash mixing speed and settling time of $12 \mathrm{mg} / \mathrm{L}, 9,300 \mathrm{rpm}$ and $2 \mathrm{~h}$, respectively. Under optimum conditions, the removal efficiency was 90.71 and $76.54 \%$ for residual turbidity and COD.
\end{abstract}

Key Words: Poly-diallyldimethyl ammonium chloride, Taguchi method, Experimental design, ANOVA, Zeta potential.

\section{INTRODUCTION}

Coagulation-flocculation process is an important environmental protection technology with a wide range of application in water or wastewater treatment facilities. It is considered as a viable option for the treatment of surface water to remove suspended solids, colloidal particles and natural organic matter producing drinking water; as well as heavy loaded, toxic or hazardous wastewaters, including landfill leachate ${ }^{1-3}$. Application of the coagulation/flocculation process combined with other appropriate physico-chemical or biological treatment processes such as ozonation, photo-oxidation or submerged biological filters, results in enhanced efficiency during wastewater treatment ${ }^{4}$. However, the process largely depends on the role of coagulants/flocculants. In addition, because of economic development, increasing volumes of wastewater generated daily contain various chemical substance and solid particles, which are a serious threat to human health. Therefore, there is a need for improving the treatment efficiency during the coagulation-flocculation process to address this problem ${ }^{5-7}$.

There are two major mechanisms involved in the flocculation of charged particles; charge neutralization and chain bridging ${ }^{8,9}$. Depending on the mechanism involved, either repulsion forces between similar charged particles are reduced and thus natural attractive forces bring about destabilization, or repulsive forces are overcome by polymer chain bridging ${ }^{10}$. Ideally, a coagulation/flocculation test procedure should simulate the operation of a full-scale unit and be capable of predicting plant performance based on laboratory trials. However, due to various reasons, such prediction is difficult to achieve and a more realistic aim is to establish optimal chemical conditions in laboratory trials, which correspond closely to that giving optimum plant performance ${ }^{11}$.

The application of synthetic polyelectrolytes in water and wastewater treatment has been known since the late 1950's. On the other hand, polyelectrolyte's coagulants used in water/ wastewater treatment are high molecular weight, organic compounds that have a strong tendency to be adsorbed on the surface of particles in an aqueous suspension ${ }^{12-17}$. They are normally applied in conjunction with inorganic metallic coagulants such as iron(III) chloride or alum ${ }^{18}$. Polymer flocculants are beneficial in coping with the problem of slow settling flocs in low temperature coagulation or in treating soft coloured waters, where they improve the settle-ability and increase the toughness of flocs ${ }^{17}$.

Organic polyelectrolytes are typically hydrophilic colloids and contain functional groups that may be ionizable. Upon dissociating, the polymer molecules become charged; either 
positively or negatively and are thus referred to as cationic or anionic polyelectrolyes, respectively ${ }^{14}$. Polyelectrolytes have been the subject of extensive investigation for several decades and still continue to be an active area of research in diverse fields. Cationic poly-quaternary ammonium salt poly-diallyldimethyl ammonium chloride (PDADMAC) is a water-soluble polymer and interacts with anionic high and low molecular weight substances present in aqueous solution ${ }^{5,19,20}$. Furthermore, PDADMAC is a large-scale product with many industrial and environmental applications, e.g., as a retention aid, flocculant, antistatic, dye fixation, electro-conductive coating and biocides ${ }^{21,22}$.

The Taguchi balance orthogonal array approach is mostly used in the industrial environment, but can also be used in scientific research for finding factors that are most important in achieving useful goals ${ }^{23-25}$. For parameter optimization problem with a given computable objective function, the Taguchi method of experimental design can rapidly optimize the varying factors to get a desired outcome. It involves an analysis that reveals which of the factors are most effective in reaching the goal and the direction in which these factors should be adjusted to improve the results ${ }^{23,26}$. A fractional factorial design such as the Taguchi method uses a subset of the possible value combination to reduce the time for conducting experiments while taking advantage of full factorial method ${ }^{27}$.

In this research, PDADMAC was synthesized using the Taguchi method of design of experiments and optimum conditions for synthesis of PDADMAC were determined. The key factors selected for investigation in the synthesis of PDADMAC were synthesis temperature, mass of the initiator and synthesis time with an objective of finding the combination of factors to achieve high turbidity removal in wastewater. The coagulation behaviour of PDADMAC was assessed by studying the turbidity and chemical oxygen demand (COD) removal efficiency on wastewater.

\section{EXPERIMENTAL}

All reagents used in this study were analytical grade except diallydimethyl ammonium chloride (DADMAC), which was technical grade. The other reagents used were 2,2'azobis[2-(2-imidazolin-2-yl)propane] dihydrochloride $\left(\mathrm{V}_{\mathrm{a} 044}\right)$, sodium hydroxide $(\mathrm{NaOH})$ and Hydrochloric acid $(\mathrm{HCl})$. All aqueous solutions and standard solutions were prepared with de-ionized water.

Sampling: The wastewater was collected from the sewer system of Chongqing University and its characteristics are presented in Table-1. After filtering to remove large particles, the samples were used for coagulation/flocculation experiments immediately.

TABLE-1

CHARACTERISTICS OF WASTEWATER SAMPLE

\begin{tabular}{cc}
\hline Parameter & Range \\
\hline $\mathrm{pH}$ & $7.32-8.47$ \\
$\mathrm{COD}_{\mathrm{cr}}(\mathrm{mg} / \mathrm{L})$ & $90-290$ \\
Turbidity $(\mathrm{NTU})$ & $60-100$ \\
\hline
\end{tabular}

Preparation of PDADMAC: PDADMAC investigated in this study was a coagulant/flocculant prepared in the laboratory. The steps for its preparation were as follows:
A three-necked, round-bottom flask $(250 \mathrm{~mL})$ was used as the reaction vessel. Firstly, $20 \mathrm{~g}$ of diallyldimethyl ammonium chloride monomer was added to a reaction vessel followed by the initiator, $\mathrm{V}_{\mathrm{a} 044}(0.05-0.2 \mathrm{~g})$ to prepare the monomer phase. Then, de-ionized water $(6-12 \mathrm{~mL})$ used to rinse the glassware after transferring the monomer and initiator to the reaction vessel was added to the mixture. The mixture was purged using nitrogen gas with agitation to remove oxygen for $20 \mathrm{~min}$. Lastly, the reaction vessel was heated with a thermostatic controlled water to different temperatures $\left(40-70{ }^{\circ} \mathrm{C}\right)$, which was maintained for a specific time (5-8 h). The resulted polymer solution was cooled to room temperature before storage.

Characterization of composite coagulant: Sample of PFS and $\mathrm{PFPD}_{3}$ was dried at $55^{\circ} \mathrm{C}$ in an oven and mixed with potassium bromide $(\mathrm{KBr})$ and the respective pellet, suitable for FT-IR analysis, was prepared. A FT-IR spectrum was recorded in the range of $4000-400 \mathrm{~cm}^{-1}$. In addition, SEM examined the surface morphology of the coagulants.

Coagulation-flocculation experiments: The coagulation/ flocculation experiments were carried out using a programcontrolled jar test apparatus (ZR4-6, Zhongrun Water Industry Technology Development Co. Ltd., China) at room temperature. $500 \mathrm{~mL}$ of wastewater was transferred into a beaker and the initial $\mathrm{pH}$ was adjusted to the set value using $0.5 \mathrm{~mol} / \mathrm{L}$ $\mathrm{HCl}$ and $\mathrm{NaOH}$. Under rapid mixing with the set agitation speed of $300 \mathrm{rpm}$, predetermined amount of coagulant was dosed. After $2 \mathrm{~min}$, the speed was changed to a slow speed of $50 \mathrm{rpm}$ for $10 \mathrm{~min}$. Lastly, after quiescent settling of $2 \mathrm{~h}$, the clarified wastewater was extracted from $2 \mathrm{~cm}$ below the surface for measurement of residual turbidity and COD.

\section{RESULTS AND DISCUSSION}

Optimum synthesis conditions of PDADMAC: The factors investigated for synthesizing PDADMAC using the Taguchi method were synthesis temperature, mass of initiator, volume of de-ionized water and the synthesis time. In accordance with the Taguchi method, the standard orthogonal array $\mathrm{L}_{16}$ with only 16 experimental runs was sufficient.

Fig. 1(a-d) shows the main effect plot of the four factors at four levels on the mean turbidity removal efficiency. Fig. 1(a) shows an increase in the mean turbidity removal efficiency as the temperature increased from 40 to $50{ }^{\circ} \mathrm{C}$, indicating a positive effect. However, the mean turbidity removal efficiency decreased from 50 to $60^{\circ} \mathrm{C}$, signifying a negative effect, while from 60 to $70{ }^{\circ} \mathrm{C}$ there was no effect in the mean turbidity removal efficiency. Therefore, $50{ }^{\circ} \mathrm{C}$ was selected as the optimum synthesis temperature. Fig. 1(b) shows an increase in the mean turbidity removal efficiency as the mass of the initiator increased from 0.05 to $0.15 \mathrm{~g}$, signifying a positive effect. Nevertheless, the mean turbidity removal efficiency almost stabilized as mass of the initiator increased from 0.15 to $0.2 \mathrm{~g}$, indicating a negligible effect. Taking into account the cost implications of the initiator, $0.15 \mathrm{~g}$ was adopted as the optimum value for the mass of the initiator required for synthesizing PDADMAC.

Fig. 1(c) shows an increase in the mean turbidity removal efficiency as the volume of de-ionized water increased from 6 to $8 \mathrm{~mL}$ and from 8 to $10 \mathrm{~mL}$, indicating a positive effect. 
A

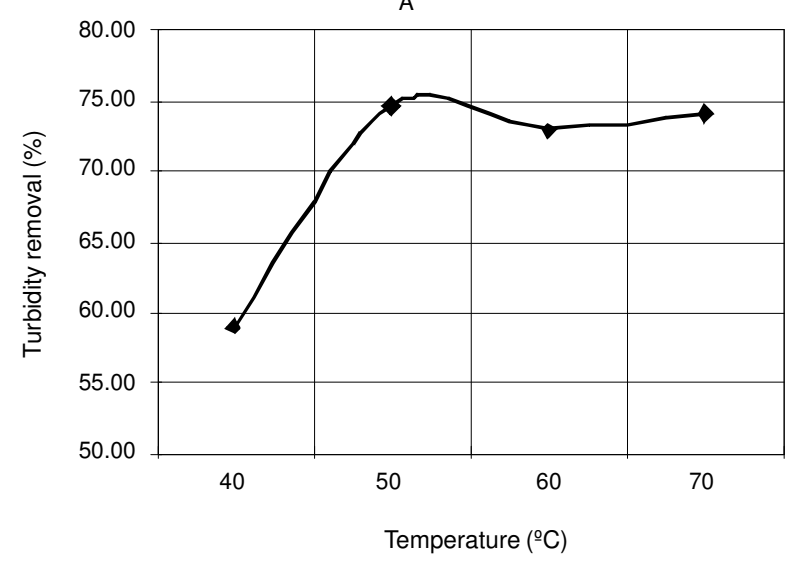

B

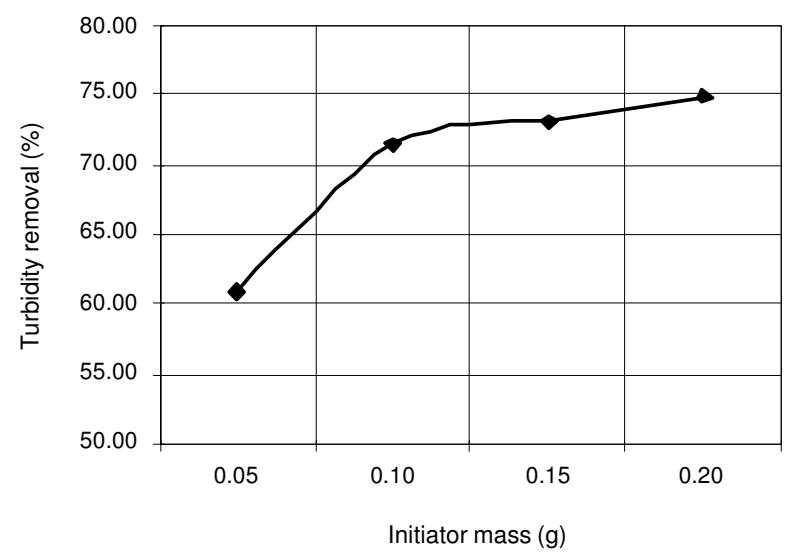

C

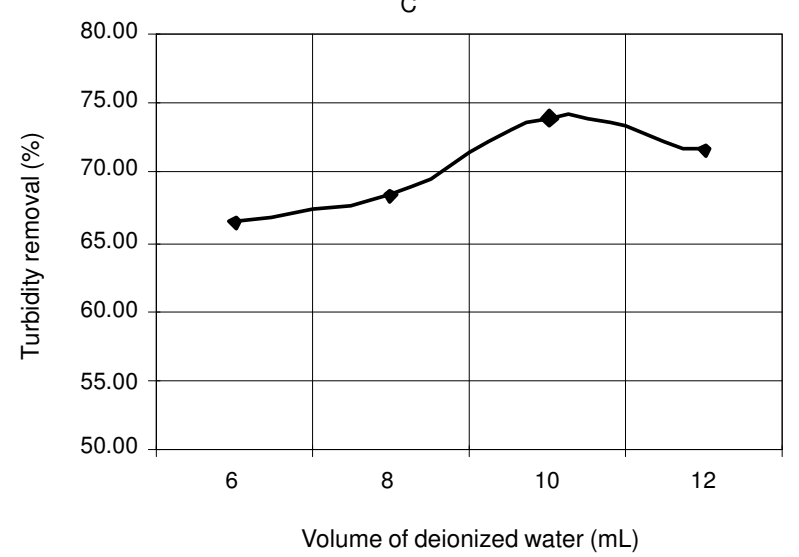

D

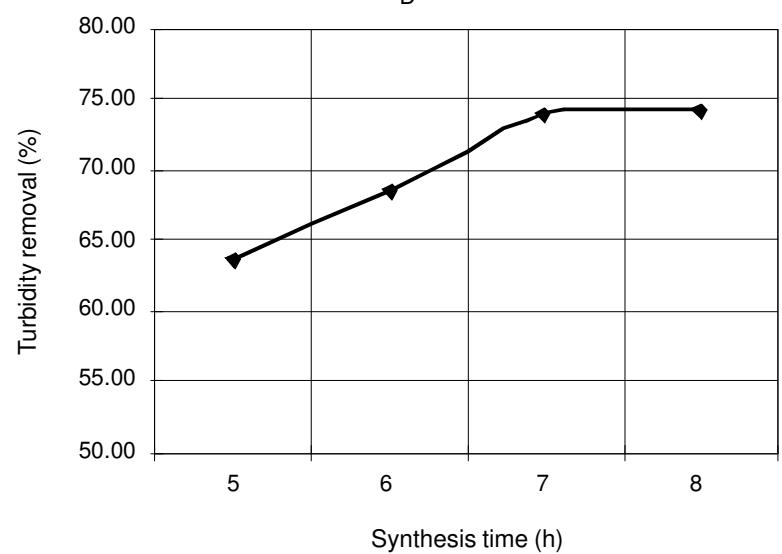

Fig. 1. Main effect plot for (A) temperature, (B) mass of initiator, (C) volume of water and (D) synthesis time on mean turbidity removal
However, the mean turbidity removal efficiency decreased as the volume of de-ionized water increased from 10 to $12 \mathrm{~mL}$, signifying a negative effect. As a result, $10 \mathrm{~mL}$ was adopted as the optimum value for volume of de-ionized water required for synthesizing PDADMAC. Furthermore, Fig. 1(d) shows an increase in the turbidity removal efficiency as the synthesis time increased from 5 to $8 \mathrm{~h}$, indicating a positive effect in the mean turbidity removal efficiency. Therefore, $8 \mathrm{~h}$ was adopted as the optimum synthesis time. The combination of factors for achieving the best turbidity removal efficiency according to the Taguchi design for synthesis temperature, mass of the initiator, volume of de-ionized water and synthesis time were $50{ }^{\circ} \mathrm{C}, 0.15 \mathrm{~g}, 10 \mathrm{~mL}$ and $8 \mathrm{~h}$, respectively.

Statistical analysis: The main objective of the analysis was to determine the influence of every parameter on the variance of the results, regarding the total variance of all parameters.

Table- 2 shows the ANOVA results performed on the experimental data. According to Table-2, the synthesis temperature had the most significant influence on the turbidity removal efficiency, followed by the mass of initiator, synthesis time and volume of de-ionized water. Therefore, the synthesis temperature and the mass of the initiator played a key role on the synthesis of the PDADMAC. The experimental error was $12.76 \%$, but that does not mean the results are not valid. This indicates there were other influential factors that were not included in the initial experimental design.

Effect of coagulant dosage on coagulation/flocculation: The purpose of this experiment was to investigate the effect of coagulant dose on the coagulation-flocculation process at wastewater initial $\mathrm{pH}$ value of 7 , using agitation speed of 300 $\mathrm{rpm}$ and settling time of $2 \mathrm{~h}$. In addition, charge neutralization capacity of the coagulant was measured using zeta potential analyzer. The wastewater initial $\mathrm{pH}$ was adjusted using 0.5 $\mathrm{mol} / \mathrm{L} \mathrm{HCl}$ and $\mathrm{NaOH}$.

Fig. 2 shows the effect of the coagulant dosage on the coagulation-flocculation efficiency. The result indicated that the coagulant dosage largely influences both the turbidity and COD removal efficiency. With an increase in the coagulant dosage, turbidity removal efficiency increased until a dosage of $12 \mathrm{mg} / \mathrm{L}$, but increasing the dosage above this point, turbidity removal efficiency decreased. The main reason for the decrease is the overdosing effect. At lower dosages, the amount of polymer to form adequate bridging links between particles is insufficient, while with excess polymer, there is no longer enough bare particle surface available for attachment of segments resulting in particle destabilization ${ }^{28}$. The results showed that the best turbidity removal efficiency of $86.56 \%$ was achieved at an optimum coagulant dosage of $12 \mathrm{mg} / \mathrm{L}$, while dosages above $12 \mathrm{mg} / \mathrm{L}$ led to a decrease in the removal efficiency. In addition, Fig. 2 shows that for COD removal, as the dosage increases, the removal efficiency increased until a dosage of $10 \mathrm{mg} / \mathrm{L}$ and then decreased with increasing dosage. However, despite a slight decrease in the COD removal from $10-12 \mathrm{mg} / \mathrm{L}$, it ensured an increase in the turbidity removal efficiency. Therefore, the adopted optimum dosage was 12 $\mathrm{mg} / \mathrm{L}$ at which the turbidity and COD removal efficiencies were 86.56 and $60.76 \%$. 


\begin{tabular}{lccccc}
\hline \multicolumn{5}{c}{ TABLE-2 } \\
\multicolumn{1}{c}{ Factors } & Degree of freedom & Som of squares & Mean square & $F^{-}$ratio & Percentage contribution \\
\hline Temperature & 3 & 668.19 & 222.73 & 2.90 & 36.97 \\
Initiator mass & 3 & 474.50 & 158.17 & 2.06 & 26.25 \\
Water volume & 3 & 136.36 & 45.45 & 0.59 & 7.54 \\
Synthesis time & 3 & 297.85 & 99.28 & 1.29 & 16.48 \\
Error & 3 & 230.69 & 76.90 & - & 12.76 \\
Total & 15 & 1807.59 & - & - & 100 \\
\hline
\end{tabular}

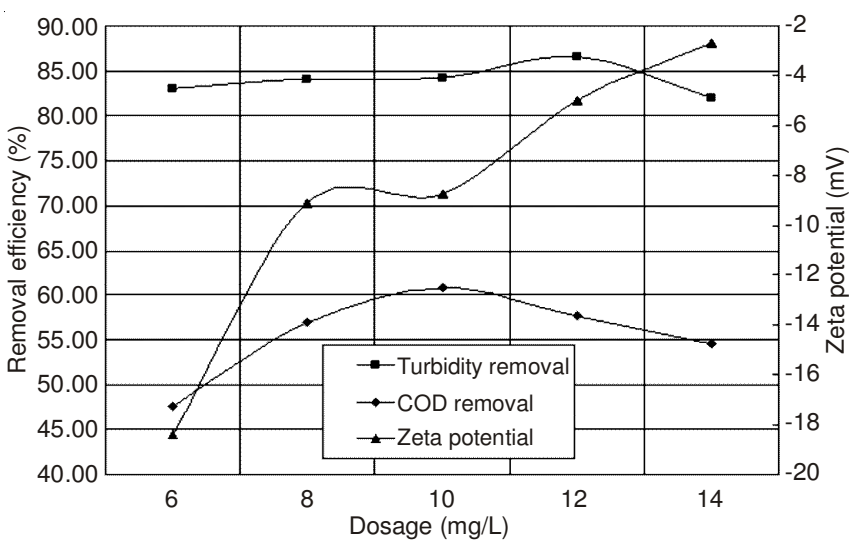

Fig. 2. Effect of coagulant dosage on turbidity and removal efficiency of COD and zeta potential measurements

Fig. 2 shows that as the coagulant dosage increased, zeta potential increased significantly to values close to the point of zero charge $(0 \mathrm{mV})$ at a dosage of $14 \mathrm{mg} / \mathrm{L}$. However, zeta potential was negative for the dosage range under investigation. The zeta potential value at the optimum coagulant dosage of $12 \mathrm{mg} / \mathrm{L}$ was $-4.97 \mathrm{mV}$, which was below the point of zero charge. Therefore, zeta potential results indicated that although the surface charge may have overall neutrality, there are patches or islands of positive charge between regions of uncoated, negative charged surface ${ }^{9}$. Therefore, charge neutralization (electrostatic patch effect) mechanism played a role in the coagulation-flocculation process.

Effect of wastewater initial pH value on coagulation/ flocculation: The aim of this experiment was to investigate the influence of wastewater initial $\mathrm{pH}$ value on coagulationflocculation efficiency at predetermined optimum coagulant dosage of $12 \mathrm{mg} / \mathrm{L}$, using agitation speed of $300 \mathrm{rpm}$ and settling time of $2 \mathrm{~h}$. The wastewater initial $\mathrm{pH}$ was adjusted in the range 5-9 using $0.5 \mathrm{~mol} / \mathrm{L} \mathrm{HCl}$ and $\mathrm{NaOH}$.

Fig. 3 shows the influence of wastewater initial $\mathrm{pH}$ on the coagulation-flocculation efficiency. The result indicated that the wastewater initial $\mathrm{pH}$ largely influences both the turbidity and COD removal efficiency. With an increase of $\mathrm{pH}$ from 5 to 9 , the turbidity removal efficiency increased from 80.43 to $91.06 \%$. Any further increase in initial $\mathrm{pH}$ above 9 resulted in small flocs being formed in the wastewater sample before addition of the coagulant. Therefore, the adopted optimum value for turbidity removal was at $\mathrm{pH} 9$. The results showed that the alkaline conditions enhance the coagulation/flocculation efficiency while, on the contrary; acidic conditions were not conducive for the coagulation/flocculation efficiency. In addition, Fig. 3 shows that for COD removal, as the wastewater initial $\mathrm{pH}$ increased from 5 to 8 , the removal efficiency increased from 13.22 to $74.71 \%$ and then decreased with increasing $\mathrm{pH}$

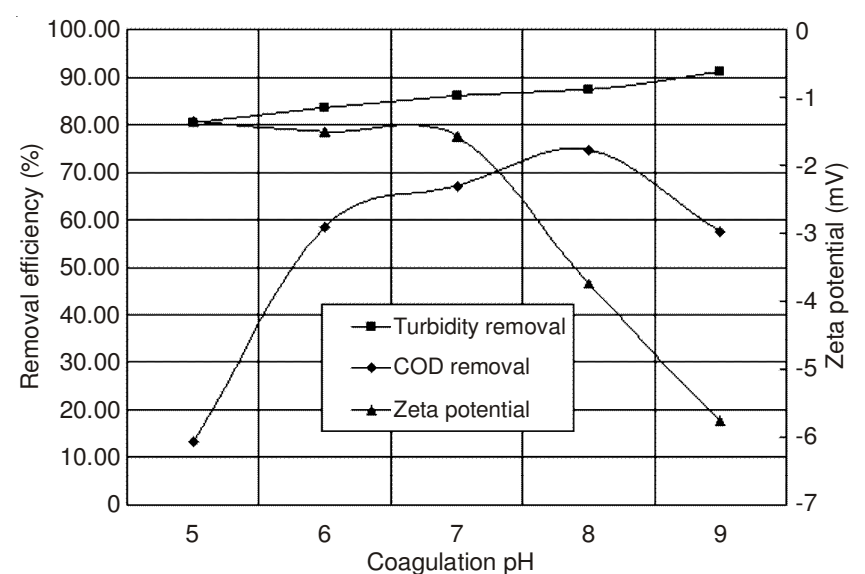

Fig. 3. Effect of wastewater initial $\mathrm{pH}$ on turbidity and removal efficiency of COD and zeta potential measurements

value. However, considering the turbidity removal efficiency, the adopted optimum $\mathrm{pH}$ value was 9 at which the turbidity and COD removal efficiencies were 91.06 and $57.47 \%$. Furthermore, Fig. 3 shows that as the wastewater initial pH increased, zeta potential decreased slightly up to $\mathrm{pH} 7$ while above $\mathrm{pH} 7$ there was a significant decrease in the surface charge of particles. Therefore, in the acid region, the zeta potential value were almost constant with a slight decrease while in the alkaline conditions there was a sharp decrease in the zeta potential value. This indicated that with an increase in $\mathrm{pH}$ value, the surface charge of the particles decreased presenting a better coagulation/flocculation performance.

Effect of flash mixing speed on coagulation/flocculation: Flash mixing speed is one of the important factors in achieving higher flocculation efficiency during the coagulation-flocculation process. In this experiment, the effect of flash mixing speeds between 150-350 rpm on the coagulation-flocculation process was investigated at predetermined coagulant dosage and wastewater initial $\mathrm{pH}$ value of $12 \mathrm{mg} / \mathrm{L}$ and 9, respectively. The supernatant was allowed to settle for $2 \mathrm{~h}$ before measurement of residual turbidity. The wastewater initial $\mathrm{pH}$ was adjusted using $0.5 \mathrm{~mol} / \mathrm{L} \mathrm{HCl}$ and $\mathrm{NaOH}$.

Fig. 4 shows the influence of flash mixing speed on the coagulation-flocculation process. The result indicated an increase in the turbidity removal efficiency as the flash mixing speed increased. However, above the flash mixing speed of $300 \mathrm{rpm}$, there was no noticeable change in the turbidity removal efficiency. In addition, Fig. 4 showed that for COD removal, as the flash mixing speed increased, the removal efficiency increased until $300 \mathrm{rpm}$ and then decreased with increasing flash mixing speed. Therefore, the adopted optimum flash mixing speed was $300 \mathrm{rpm}$ at which the turbidity and COD removal efficiencies were 90.49 and $76.54 \%$, respectively. 


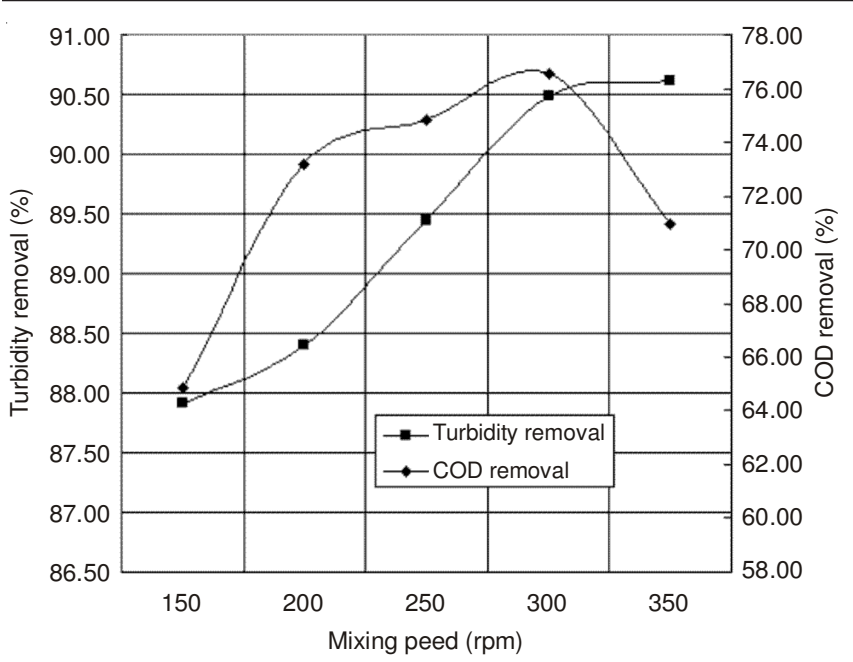

Fig. 4. Effect of flash mixing speed on turbidity and removal efficiency of COD

Effect of settling time on coagulation/flocculation: Settling time influences the quality of the treated water after the coagulation-flocculation process. Therefore, the purpose of this experiment was to investigate the influence of settling time between $0.5 \mathrm{~h}$ and $150 \mathrm{~min}$ on coagulation-flocculation efficiency at predetermined optimum coagulant dosage, wastewater initial $\mathrm{pH}$ and flash mixing speed of $12 \mathrm{mg} / \mathrm{L}, 9$ and 300 rpm respectively. The wastewater initial $\mathrm{pH}$ was adjusted using $0.5 \mathrm{~mol} / \mathrm{L} \mathrm{HCl}$ and $\mathrm{NaOH}$.

Fig. 5 shows the influence of the flash mixing speed on the turbidity and COD removal efficiency. It shows that as the settling time increases from 30 to $90 \mathrm{~min}$, there was a slight increase in the turbidity removal efficiency. However, after 90 min the removal efficiency almost stabilized around $90.70 \%$ with slight fluctuation, indicating no further improvement. In addition, Fig. 5 shows that for COD removal, as the settling time increases, the removal efficiency increased until $2 \mathrm{~h}$, with a decrease thereafter. However, despite the best turbidity removal achieved at $90 \mathrm{~min}$, there was an increase in the COD removal efficiency as the settling time increased from 90 to $120 \mathrm{~min}$. Therefore, the adopted optimum settling time was $2 \mathrm{~h}$ at which the turbidity and COD removal efficiencies were 90.62 and $53.46 \%$, respectively.

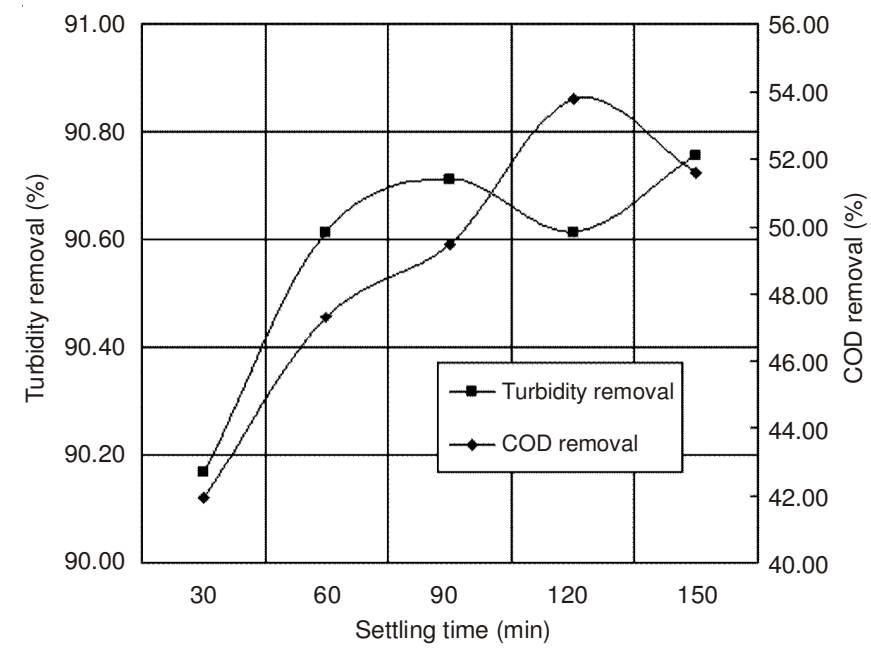

Fig. 5. Effect of settling time on turbidity and removal efficiency of COD
Lastly, the coagulation experiments were carried out at predetermined optimum conditions of $12 \mathrm{mg} / \mathrm{L}, 9,300 \mathrm{rpm}$ and $2 \mathrm{~h}$ for coagulant dosage, $\mathrm{pH}$ value, flash mixing speed and settling time, respectively. Under optimum conditions, the turbidity and COD removal efficiencies were 90.71 and $76.54 \%$, respectively.

Characterization of PDADMAC: FT-IR and SEM instruments were used to analyze and characterize the structure and morphology of PDADMAC.

FTIR spectral analysis: The molecular structure of the synthesized PDADMAC was investigated using the FTIR in the range of $4000-400 \mathrm{~cm}^{-1}$ with $\mathrm{KBr}$ as dispersant.

Fig. 6 shows the FTIR spectra of PDADMAC synthesized using $\mathrm{V}_{\mathrm{a} 044}$ as initiator. It shows characteristic peaks at 1640 and $1465 \mathrm{~cm}^{-1}$ that were assigned to the stretching vibrations of $\mathrm{NR}_{4}$ and $\mathrm{C}\left(\mathrm{CH}_{3}\right)_{2}{ }^{+}$, respectively. The peaks at 1380 and 1457 $\mathrm{cm}^{-1}$ were assigned to the symmetrical $\mathrm{C}-\mathrm{H}$ bending. In addition, the peak at $1136 \mathrm{~cm}^{-1}$ corresponds to the stretching of the C-N bond ${ }^{29,30}$. However, absorption peaks at 2972, 2932 and $2876 \mathrm{~cm}^{-1}$ resulting from the $\mathrm{C}-\mathrm{H}$ stretching vibration of $-\mathrm{CH}_{3}$ and $-\mathrm{CH}_{2}$ - were weak in the synthesized PDADMAC.

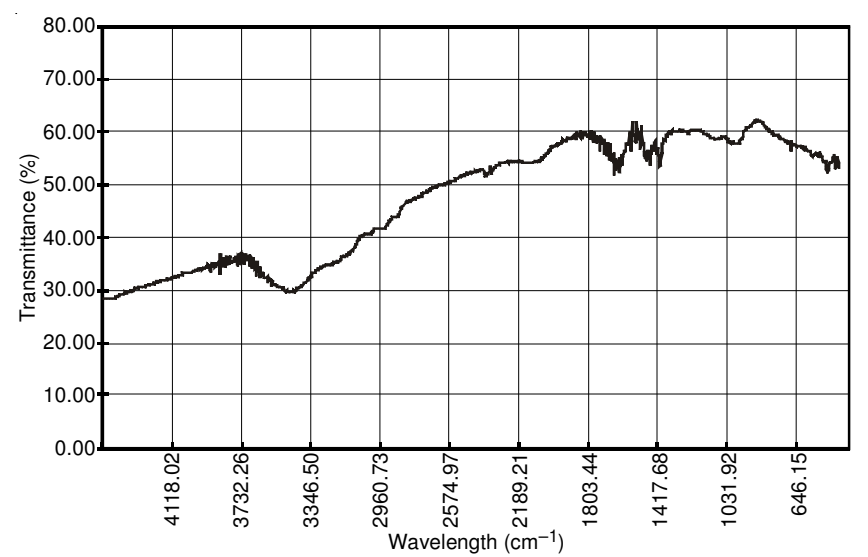

Fig. 6. FTIR spectral analysis of PDADMAC

SEM images: The surface morphology of DADMAC was analyzed using a scanning electron microscopy. Fig. 7(a-b) shows the surface morphology of PDADMAC as irregular mesh structure with a few cavities. The bigger and irregular hole in the mesh structure is good to play the bridge role for the flocculants. In addition, PDADMAC has a high positive charge density and is therefore more effective in neutralizing the negative charge on suspended colloid particles and natural organic materials during the coagulation process. Therefore, PDADMAC had good charge neutralization and adsorption/ bridging coagulation-flocculation mechanisms.

\section{Conclusion}

In this paper, the Taguchi method of design of experiments was applied in synthesizing PDADMAC and determining optimum conditions. In addition, ANOVA determined the influence of the factors on the turbidity removal efficiency. The evaluation of the PDADMAC treatment efficiency was determined by measuring both the reduction of residual turbidity and chemical oxygen demand (COD). From the investigations, we came up with the following conclusions: The combination of factors selected as optimum conditions 


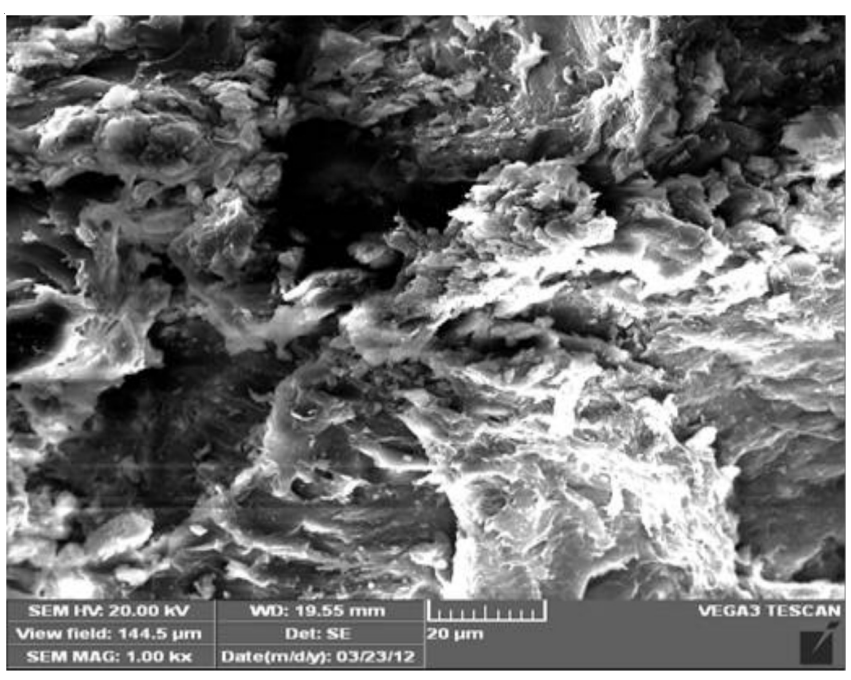

(a) SEM photographs for PDADMAC at $20 \mu \mathrm{m}$

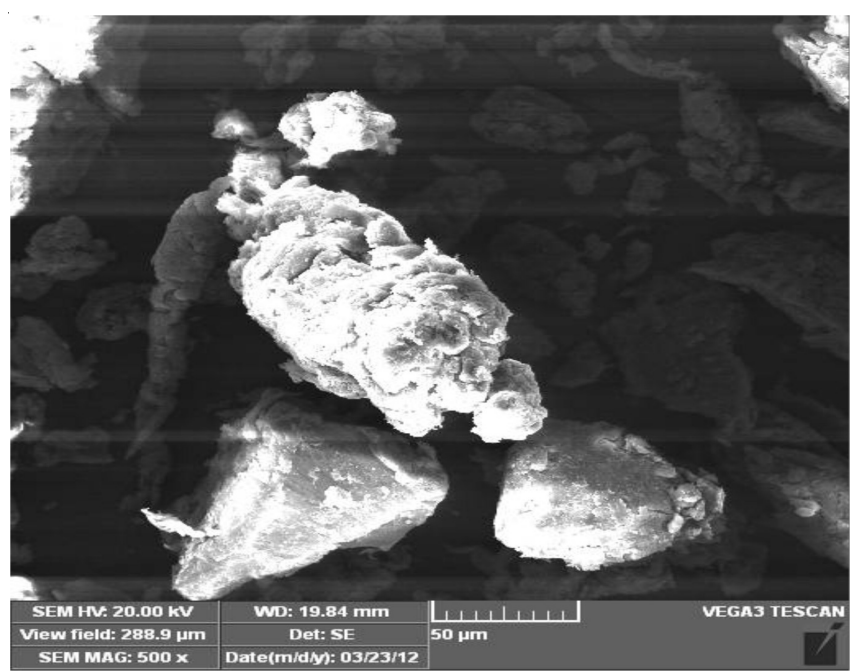

(b) SEM photographs for PDADMAC at $50 \mu \mathrm{m}$

Fig. 7. SEM photographs for PDADMAC at (a) $20 \mu \mathrm{m}$ and (b) $50 \mu \mathrm{m}$

for synthesis of PDADMAC using the Taguchi method for synthesis temperature, mass of the initiator, volume of deionized water and synthesis time were $50{ }^{\circ} \mathrm{C}, 0.15 \mathrm{~g}, 10 \mathrm{~mL}$ and $8 \mathrm{~h}$, respectively. The ANOVA results showed that the synthesis temperature had the most significant influence on the turbidity removal efficiency, followed by the mass of initiator, synthesis time and volume of de-ionized water. The optimum conditions for coagulation-flocculation were $12 \mathrm{mg} / \mathrm{L}$, 9, $300 \mathrm{rpm}$ and $2 \mathrm{~h}$ for dosage, $\mathrm{pH}$ value, flash mixing speed and settling time respectively. The turbidity and COD removal efficiency at optimum conditions were 90.71 and $76.54 \%$, respectively. From the results, the influence of $\mathrm{pH}$ value on the coagulation/flocculation process indicated that the alkaline conditions enhanced the process, while acidic conditions were not conducive for the process.

\section{ACKNOWLEDGEMENTS}

The authors are grateful for the financial support provided by the National Natural Science Foundation of China (Project No. NSFC, 51078366, 20777095), the National Science and Technology major water special project in water pollution control and governance (Project No.2009ZX07424-004), Ministry of Environmental Protection and Housing and Urban Rural Development of People's Republic of China.

\section{REFERENCES}

1. B.Y. Gao, Y. Wang, Q.Y. Yue, J.C. Wei and Q. Li, Sep. Purif. Technol., 62, 544 (2008).

2. P.A. Moussas and A.I. Zouboulis, Sep. Purif. Technol., 63, 475 (2008).

3. W. Brostow, H.E. Hagg-Lobland, S. Pal and R.P. Singh, J. Mater. Edu., 31157 (2009).

4. Y. Wang, B.Y. Gao, Q.Y. Yue, J.C. Wei and Q. Li, Chem. Eng. J., 142, 175 (2008)

5. H.Z. Zhao, Z.K. Luan, B.Y. Gao and Q.Y. Yue, J. Appl. Polym. Sci., 84, 335 (2002)

6. A.I. Zouboulis and P.A. Moussas, Desalination, 224, 307 (2008).

7. H.L. Zheng, G. Zhu, S. Jiang, T. Tshukudu, X. Xiang, P. Zhang and Q. He, Desalination, 269, 148 (2011).

8. R. Subramanian, S. Zhu and R.H. Pelton, Colloid. Polym. Sci., 277, 939 (1999).

9. B. Bolto and J. Gregory, Water Res., 41, 230 (2007).

10. D. Bauer, E. Killmann and W. Jaeger, Colloid. Polym. Sci., 276, 698 (1998).

11. T. Tripathy and B.R. De, J. Phys. Sci., 10, 93 (2006).

12. G.U. Bhaskar and S.K. Gupta, Water, Air Soil Pollut., 35, 251 (1987).

13. B.A. Bolto, Prog. Polym. Sci., 20, 987 (1995).

14. E.E. Chang, P.C. Chiang, S.H. Chao and C.H. Liang, Chemosphere, 39, 1333 (1999).

15. D. Avci, N. Mol and L. Dagasan, Polym. Bull., 48, 353 (2002).

16. H. Mahdavi, E. Haghani and B. Malakian, React. Funct. Polym., 66, 1033 (2006).

17. S. Ondaral, M. Usta, M. Gumusderelioglu, N. Arsu and D.K. Balta, J. Appl. Polym. Sci., 116, 1157 (2010).

18. J.C. Wei, B.Y. Gao, Q.Y. Yue and Y. Wang, Chem. Eng. J., 151, 176 (2009).

19. S. Francis, L. Varshney and S. Sabharwal, Eur. Polym. J., 43, 2525 (2007).

20. W. Jaeger, J. Bohrisch and A. Lascheesky, Prog. Polym. Sci., 35, 511 (2010).

21. Y. Zhang, M. Yi, J. Ren, M. Zhai and H. Ha, Macromol. Res., 11, 146 (2003).

22. B.H. Tian, B. Fan, X.J. Peng and Z.K. Luan, J. Environ. Sci., 17, 798 (2005).

23. D. Liu and Y. Cai, IEEE Trans. Power Syst., 20, 2006 (2005).

24. B. Berginc, Z. Kampus and B. Sustarsic, J. Achieve. Mater. Manuf. Eng., 15, 63 (2006).

25. M.P. Elizalde-Gonzalez and L.E. Garcia-Diaz, Chem. Eng. J., 163, 55 (2010).

26. S. Ghammamy and M.R. Baghy, J. Chem. Crystallogr., 38, 907 (2008).

27. S.S. Madaeni and S. Koocheki, Chem. Eng. J., 119, 37 (2006).

28. G. Zhu, H.L. Zheng, Z. Zhang, T. Tshukudu, P. Zhang and X. Xiang, Chem. Eng. J., 178, 50 (2011).

29. J. Lu, X.D. Wang and C.B. Xiao, Carbohydr. Polym., 73, 427 (2008).

30. J. Chen, M. Liu, H. Liu, L. Ma, C. Gao, S. Zhu and S. Zhang, Chem. Eng. J., 159, 247 (2010). 